\title{
Generating Classifier Outputs with Fixed Diversity for Evaluating Voting Methods
}

\author{
Héla Zouari ${ }^{1}$, Laurent Heutte ${ }^{1}$, Yves Lecourtier ${ }^{1}$, and Adel Alimi ${ }^{2}$ \\ ${ }^{1}$ Laboratoire Perception, Systèmes, Information (PSI), Université de Rouen \\ 76821 Mont-Saint-Aignan, CEDEX, France \\ \{Hela.Khoufi, Laurent.Heutte, Yves.Lecourtier\}@univ-rouen.fr \\ http: //www. univ-rouen.fr/psi \\ ${ }^{1}$ Groupe de Recherche sur les Machines Intelligentes (REGIM), Université de Sfax \\ Ecole National des ingénieurs, BP W, Sfax, 3038, Tunisie \\ Adel.Alimi@ieee.org
}

\begin{abstract}
Recently, it has been shown that for majority voting combination methods, (negatively) dependent classifiers may provide better performance compared to that obtained with independent classifiers. The aim of this paper is to analyse the performance of plurality voting according to classifier diversity (agreement). This analysis is conducted in parallel with majority voting in order to show which method is more efficient with dependent classifiers. For this purpose, we develop a new method for the artificial generation of classifier outputs with fixed individual accuracies and pair-wise agreement. A diversity measure is applied for building the classifier teams. The experimental results show that the plurality voting is less sensitive to the correlation between classifiers than majority voting. It is also more efficient in achieving the trade-off between the recognition rate and rejection rate than the majority voting.
\end{abstract}

\section{Introduction}

Voting methods, in particular plurality voting and majority voting, are widely used in multiple classifier systems. They operate on the class labels assigned by the classifiers to each pattern. Plurality voting means that the class with the most votes is chosen. In majority voting, the class that receives more than half of votes is chosen. For these voting rules, the use of independent classifiers is considered to be essential to achieve better performance $[5,6,12,14]$. However, in practice, it is difficult to obtain independent classifiers. Recently, the assumption of independence is questioned "Is independence good for combination?" [7] and some attention has been devoted to the relationship between dependency and accuracy of voting methods especially for the majority voting method. In [8], it is shown that independence is not a universal requirement and negatively dependent classifiers are more beneficial than independent classifiers. In [11], the authors derive the upper and lower limits on the majority vote accuracy with respect to individual classifier accuracy $p$ (the same for all classifiers), the number of classifiers in the pool and the pair wise dependence between the

\footnotetext{
A. Fred et al. (Eds.): SSPR\&SPR 2004, LNCS 3138, pp. 1001-1009, 2004.

(C) Springer-Verlag Berlin Heidelberg 2004
} 
classifiers. They showed that for achieving the highest improvement over $p$, all pairs of classifiers in the pool should have the same negative dependency. How will the results of the plurality voting be affected when the classifiers are dependent? Is the plurality voting more sensitive to correlation than the majority voting? To seek answers to these questions, a large number of classifier ensembles with desired diversity is needed. However, building such ensembles on real-life data is difficult. In this paper, we are interested in the use of diversity in designing classifier teams in order to examine empirically the relationship between the plurality voting and classifier diversity. The idea behind the proposed approach is to generate classifier outputs with fixed accuracies and fixed agreement. A statistical measure, chosen in advance, is used to determine the pair wise agreement between the classifiers.

The paper is organized as follows. Section 2 presents the techniques suggested to enforce diversity between classifiers. Section 3 discusses the diversity measure used to generate the pair-wise agreement. The process of generating two classifiers according to the predefined agreement measure is presented in section 4 . The case of more than two classifiers is addressed in section 5. Experimental results are reported in section 6. Conclusions are drawn in section 7.

\section{Enforcing Diversity}

Diversity among classifiers, in addition to individual performance of each classifier, is considered as one of the main factors, to explain the behaviour of combination methods. To create a consistent ensemble of classifiers, several implicit and explicit methods have been investigated in the literature. Duin [2] lists the main implicit approaches to build diverse classifiers. The principal one is to use different data representations adapted to the classifiers, for example, by using different feature vectors as inputs of classifiers $[6,13,16]$. The diversity can also be implicitly encouraged either by varying the classifier topology, the learning parameters [5] or by training each classifier on different parts of the data which is done for example by Bagging [1]. On the contrary, the aim of explicit methods is to design a set of classifiers by asserting the diversity measure in the process of building ensembles $[4,8,13,14]$. The advantage of the incorporation of diversity measure is to control a priori the diversity between the classifier outputs in order to facilitate the analysis of the combination behavior. In [8] for example, the authors propose a comprehensive study of the random generation of binary classifier outputs to evaluate the performance of majority voting. They derive formulas according to how two classifiers can be generated with specified accuracies and dependencies between them using Q statistic as a diversity measure. Based on these formulas, the authors propose an algorithm for generating multiple dependent classifiers. Lecee et al [13] have also studied the influence of correlation among classifiers to evaluate combination methods of class type. For that, teams of classifiers producing binary outputs are created according to fixed recognition rates and predefined similarity measure. 


\section{Diversity Measure}

The problems with diversity started to emerge with the attempts of measuring it [9]. Although used in many recognition problems, there is no analytic proof that a particular measure of diversity is preferable to another. The diversity measures operating on binary classifier outputs have been broadly categorized as pair-wise (measures which are calculated for each pair of classifiers in the team and then averaged) and non pairwise [9, 15]. Such measures have been studied for artificial datasets [9] or for realworld datasets [15] to analyse the correlation between diversity and majority voting performance. In [9], the authors used ten diversity measures (four averaged pair-wise measures and six non pair-wise measures). Since most of the investigated measures showed very weak correlation with majority voting performance, they recommended the pair-wise Q statistic based on the criteria that it is understandable and relatively simple to implement. To emphasize the relationship between the plurality voting and diversity as well as the difference with the majority voting, we use in this study the kappa measure $K$ to estimate the level of agreement between the classifier outputs [3]. We slightly favoured this measure for the following reasons: $K$ depends on the individual accuracies of the classifiers and has a specific value 0 for statically independent classifiers. $K$ varies between -1 and +1 . Values of $K$ close to +1 indicate that the classifiers tend to recognize the same objects correctly. If the classifiers commit errors for different objects, then $K$ takes negative values. In this paper, we assess a global agreement on correct and incorrect classification.

More specifically, let $A_{i}$ and $A_{j}$ be two classifiers providing S outputs for a Nclass classification problem. Each output is made up of input pattern $b_{s}(\mathrm{~s}=1, \ldots, \mathrm{S})$ and class labels $\mathrm{C}_{\mathrm{i}}\left(\mathrm{i}=1\right.$ to $\mathrm{N}$ ). We consider that when $A_{i}$ and $A_{j}$ propose the true class label $b_{s}$ in their outputs, they agree. If they both propose incorrect labels, they also agree. In all the other cases, they disagree. Let $p_{i}$ be the accuracy or the recognition rate of $A_{i}$ which stands for the ratio of the number of outputs in which the true class appears on the total number of outputs. We can represent the output of classifier $A_{i}$ as an S-dimensional binary vector $V_{i}=\left[v_{1, i}, \ldots, v_{S, i}\right]^{T}$ such that $v_{s, i}=$ 'c' (correct), if the output $s$ contain the true class label $b_{s}$, and $v_{s, i}=$ 'w' (wrong) otherwise. Thus $\mathrm{S} \times p_{i}$ elements of $V_{i}$ have values 'c' and $\mathrm{S} \times\left(1-p_{i}\right)$ elements have values ' $w$ '. Thus, the N-class problem is transformed into a 2-class problem depending on the presence or absence of the true class in the classifier outputs.

The pair-wise agreement between $A_{i}$ and $A_{j}$ having accuracies $p_{i}$ and $p_{j}$ can be obtained by the following function [9]:

$$
\kappa_{i, j}=1-\frac{p_{c w}+p_{w c}}{2 \bar{p}(1-\bar{p})}
$$


where $\bar{p}$ is the mean accuracy of the two classifiers, $\bar{p}=\frac{p_{i}+p_{j}}{2}$ $p_{x y}$ is the probability that $v_{s, i}=\mathrm{x}$ and $v_{s, j}=\mathrm{y}$ (see Table 1 ).

The probabilities estimated in Table 1 are obtained by: $p_{c c}=\frac{n_{c c}}{S}, p_{c w}=\frac{n_{c w}}{S}$, $p_{w c}=\frac{n_{w c}}{S}$ and $p_{w w}=\frac{n_{w w}}{S}$ with $n_{c c}+n_{c w}+n_{w c}+n_{w w}=S$

where $n_{x y}$ is the number of outputs in which the classifiers $A_{i}$ and $A_{j}$ propose or not the true class $b_{s}$.

Table 1. Diversity matrix $D M_{i j}$ representing the percentage of agreement and disagreement between two classifiers $A_{i}$ and $A_{j}$

\begin{tabular}{|l|l|l|}
\hline & $A_{j} \operatorname{correct}(\mathrm{c})$ & $A_{j}$ wrong (w) \\
\hline$A_{i} \operatorname{correct}(\mathrm{c})$ & $p_{c c}$ & $p_{c w}$ \\
\hline$A_{i}$ wrong (w) & $p_{w c}$ & $p_{w w}$ \\
\hline
\end{tabular}

\section{Output Generation for Two Classifiers}

Generating classifiers according to accuracy only is easy. However, when the diversity has to be considered, the generation is not straightforward. Recently Kuncheva and Kountchev propose a method for generating multiple classifier (binary) outputs [8]. They derive formulas according to how two classifiers can be generated with desired accuracies and dependency $\mathrm{Q}$ between them. The generation of the classifiers is realized simultaneously. Inspired by this study, we present here another possible solution based on the kappa measure. The idea is to determine a priori the relationship (agreement or disagreement) between the two classifiers through the diversity matrix $D M$ according to fixed accuracies and pre-specified agreement level and then to generate, from this matrix, the classifier outputs. The outputs of the first classifier in each team are generated by the classifier simulator developed in our previous work [18]. The generation procedure of these outputs is beyond the scope of this paper and has been discussed earlier in a separate paper [18]. Here we concentrate on the generation of the second classifier based on the outputs of the first classifier and the diversity matrix. Thus, when two classifiers $A_{1}$ and $A_{2}$ are to be simulated according to a predefined diversity level, the procedure of generation follows 3 steps: 
1. Construction of the diversity matrix $D M_{12}$ between $A_{1}$ and $A_{2}$ according to the desired accuracies $p_{1}, p_{2}$ and kappa $\kappa_{1,2}$.

2. Generation of the outputs of $A_{1}$ according to the fixed accuracy $p_{1}$.

3. Generation of the second classifier from $A_{1}$ taking into account the accuracy $p_{2}$ and the diversity matrix $D M_{12}$.

After simple algebraic manipulations using Table 1, we can express the entries $p_{c c}$, $p_{c w}, p_{w c}$ and $p_{w w}$ using $p_{1}, p_{2}, \bar{p}$ and $\kappa_{1,2}$ as:

$$
\begin{aligned}
& p_{c c}=\bar{p}\left((1-\bar{p})\left(\kappa_{1,2}-1\right)-1\right) \\
& p_{c w}=p_{1}-p_{c c} \\
& p_{w c}=p_{2}-p_{c c} \\
& p_{w w}=1-2 \bar{p}+p_{c c}
\end{aligned}
$$

After computing the elements of the diversity matrix $D M_{12}$, we generate the outputs $O_{1}=\left[o_{1,1}, \ldots, o_{1, S}\right]$ of the basic classifier $A_{1}$ according to the desired accuracy $p_{1}$ and we transform it into the binary vector $V_{1}$. Based on this vector and $D M_{12}$, we generate the vector $V_{2}$ of the classifier $A_{2}$. To do that, we should place the solutions 'c' or 'w' in the outputs of $V_{2}$. This generation depends on the number of elements $n_{c \text {. }}$ that is the number of outputs for which the first classifier provides ' $c$ ' and $n_{w}$. that is the number of outputs for which the first classifier provides ' $\mathrm{w}$ ' with $n_{c \text {. }}=n_{c \mathrm{c}}+n_{c \mathrm{w}}$ and $n_{w .}=n_{w c}+n_{w w}$. Next, we generate the vector $O_{2}$ of the classifier $A_{2}$ according to $O_{1}, V_{1}$ and $V_{2}$. This procedure is illustrated in Table 2 .

Table 2. Generating outputs of the classifier $A_{2}$ from the classifier $A_{1}$

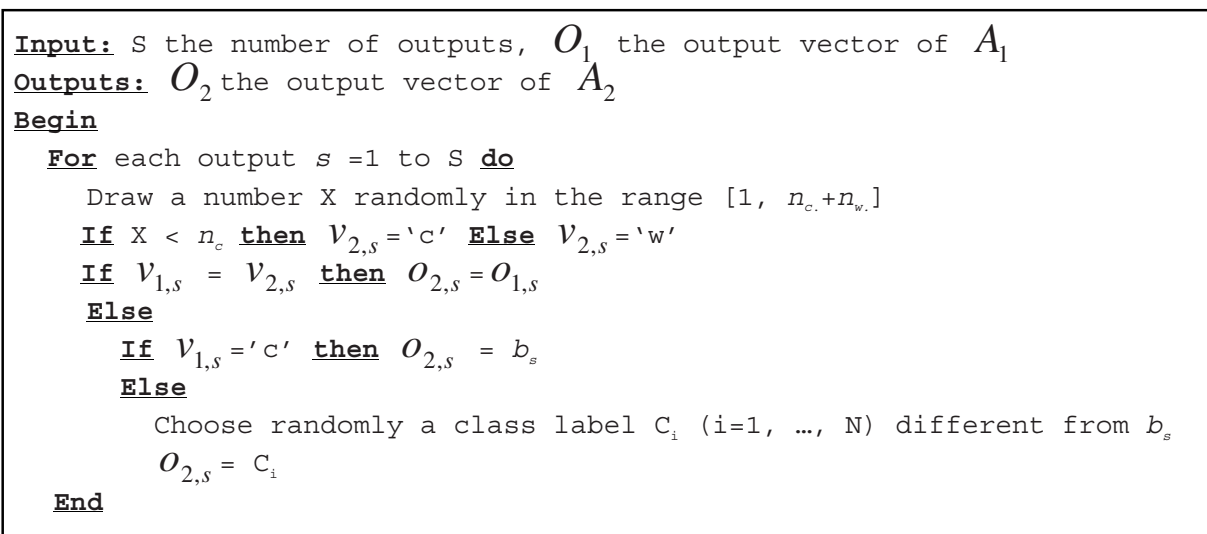




\section{The Case of More Than Two Classifiers}

The aim of the procedure described in this section is to generate automatically $\mathrm{S}$ outputs of L classifiers, for a N-class problem, which can be used for analysing the performance of voting methods. The input is the number of classes $\mathrm{N}$, the number of outputs $\mathrm{S}$, a vector with the desired individual accuracies $\boldsymbol{p}=\left[p_{1}, \ldots p_{L}\right]^{\mathrm{T}}$, and a Kappa matrix $\kappa=\left[\kappa_{i, j}\right]$, where $\kappa_{i, j}$ is the desired agreement between classifiers $A_{i}$ and $A_{j}$ (i $=1, \ldots, \mathrm{L}-1 ; \mathrm{j}=\mathrm{i}+1, \ldots, \mathrm{L})$. In the first step, the diversity matrices $D M_{i j}$ are determined for each pair of classifiers $A_{i}$ and $A_{j}$ using (2). With L classifiers, we obtain $\mathrm{L}(\mathrm{L}-1) / 2$ possible diversity matrices. The outputs of the first classifier are next generated according to the desired accuracy $p_{1}$. Starting from these outputs, outputs of the other classifiers are generated. For example, take 3 classifiers. For each output $\mathrm{s}$ ( $\mathrm{s}=1$ to $\mathrm{S}$ ), we use $o_{1, s}$ to set the output label $o_{2, s}$ of $A_{2}$ as presented in Table 2. Next, we use $o_{1, s}$ to generate the output label of $A_{3}$ according to the diversity matrices $D M_{13}$ and $D M_{23}$. Note that there is no reason to use the same order of the classifiers. The selection can be done dynamically by generating for each output $s$, a random permutation of the integers from 2 to L. This is used to pick the order in which the classifiers will be selected. This generation process is repeated S times, so that L-1 output vectors are obtained. (see Table 3 ).

Table 3. Algorithm for the generation of classifier outputs according to the fixed accuracies and pair-wise agreement $\kappa$

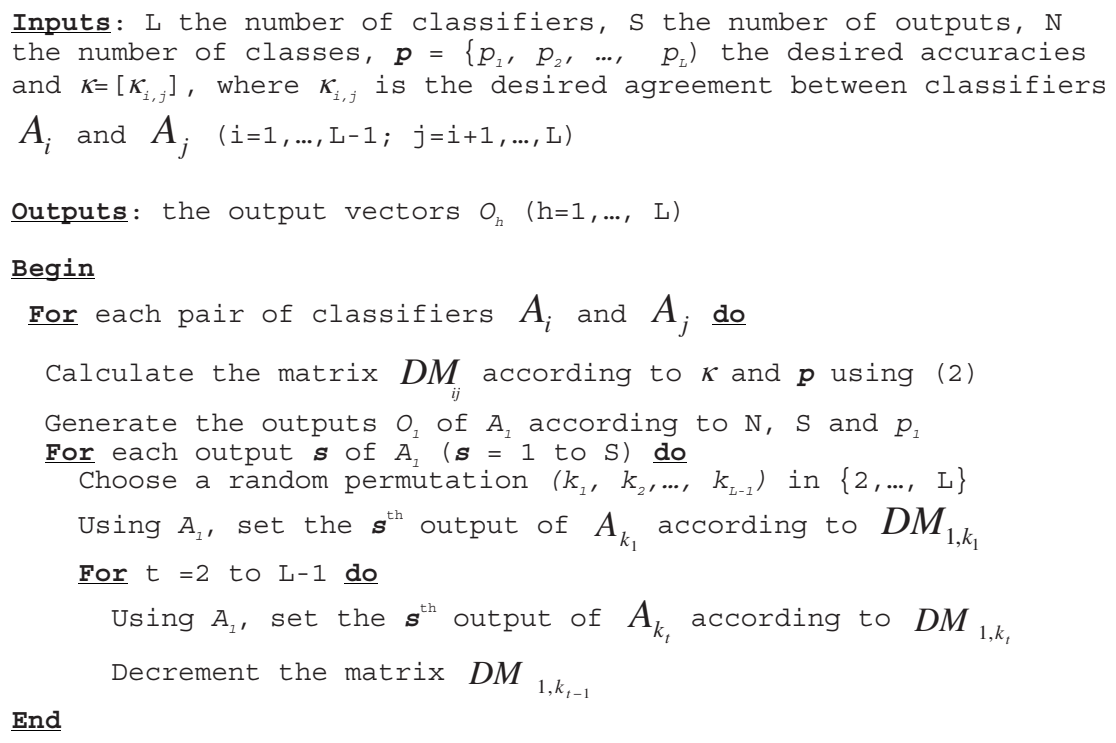


Note that there are cases where the generation of classifier ensembles with fixed diversity and accuracy are not possible (when the elements of the diversity matrix are negative). This can be explained by the fact that the kappa measure depends on the individual classifier accuracy. For two classifiers having performance equal to 0.5 , the diversity can varied between -1 and +1 . But, as the accuracy increases, the range of the diversity decreases. For example, the diversity between two classifiers with $\mathrm{p}=0.9$, vary in $[-0.1,1]$.

\section{Experimental Results}

In this section, we report two experiments aimed at examining the relationship between the performance of voting methods and diversity in classifier behavior. In these experiments, we simulate ensembles of classifiers with the same pair-wise agreement $\kappa=\{-0.4,-0.2,0,+0.2,+0.4\}$ for a 10 -class problem. For each classifier, $\mathrm{S}=10000$ outputs are generated. The goal of the first experiment is to study the effect of an additional classifier on the performance of plurality voting method. For this experiment, we simulate two classifiers with the same recognition rate $p_{1}=p_{2}$ in $\{0.5,0.6$, $0.7\}$ to which is added a third one whose recognition rate performance $p_{3}$ vary from 0.5 to 0.7 by 0.1 step. The recognition rates of the three individual classifiers and the plurality voting are shown in Figure 1. The results of Figure 1a demonstrate that the addition of the third classifier is interesting for pairs of classifiers having recognition rates higher than 0.5 . In this case, as the value of kappa measure increases (i.e. the diversity decreases), the performance of plurality voting decrease. However, the addition of the third classifier does not achieve better performance over the best individual classifier for classifiers having recognition rate equal to 0.5 , even in negatively dependent situation. Figure $1 \mathrm{~b}$ and $1 \mathrm{c}$ show that for diverse and independent classifiers, the addition of a third classifier is beneficial for combination whatever its performance. However, for kappa $>0$, the combination is not interesting.

The main goal of the second experiment is to determine which voting methods would perform best under the condition that all of the classifiers have the same accuracy $p=0.5$ (i.e. weak classifiers). Table 4 shows the results of combining five classifiers by plurality and majority voting. These results show that plurality voting outperforms majority voting for different classifier ensembles. The performance of majority voting is quite similar to the individual classifiers. The combination by this rule is not interesting in this case. Consider the combination of three classifiers with $p_{1}=p_{2}=p_{3}$ $=0.5$ as in the Figure 1a. We can see that the addition of two classifiers in the second experiment improves significantly the recognition rates of the plurality voting. The behaviour of the plurality voting becomes more stable than in the previous experiment. Another advantage of plurality voting over majority voting is its higher rejection efficiency. Plurality voting has a lower rejection rate than the majority voting. This rejection rate increases when the diversity between the classifiers decreases. The experimental results in [14] also support this conclusion when combining independent classifiers. 


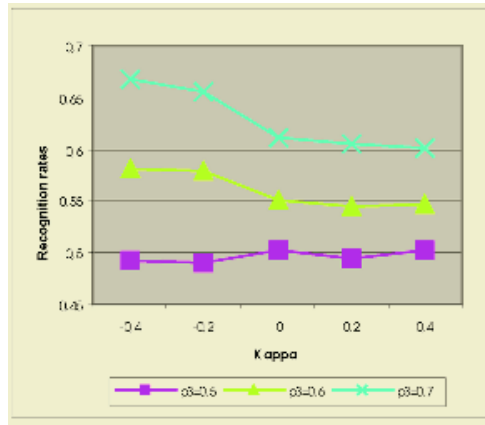

(a) $p_{1}=p_{2}=0.5$

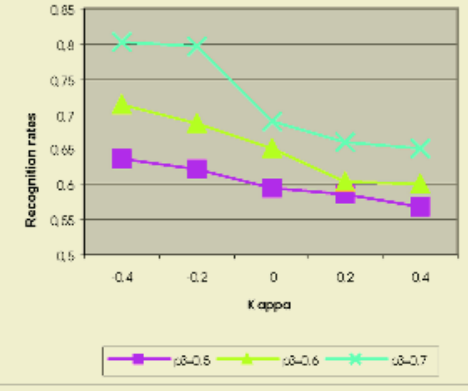

(b) $p_{1}=p_{2}=0.6$

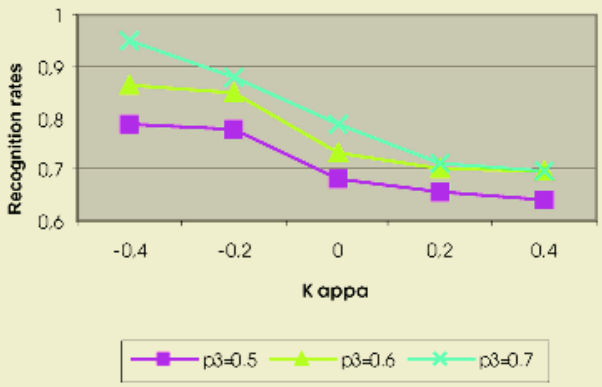

(c) $p_{1}=p_{2}=0.7$

Fig. 1 . Combination results with different performance of classifiers

Table 4. Results of combining five weak classifiers with the two combining rules

\begin{tabular}{|lllll|}
\hline & Majority voting & \multicolumn{3}{c|}{ Plurality voting } \\
\hline Diversity & Recognition rates & Rejection rates & Recognition rates & Rejection rates \\
\hline-1 & $0.504 \pm 0.001$ & $0.492 \pm 0.010$ & $0.812 \pm 0.036$ & $0.143 \pm 0.045$ \\
-0.5 & $0.501 \pm 0.004$ & $0.495 \pm 0.002$ & $0.741 \pm 0.009$ & $0.186 \pm 0.007$ \\
0 & $0.500 \pm 0.030$ & $0.494 \pm 0.004$ & $0.737 \pm 0.016$ & $0.190 \pm 0.018$ \\
0.5 & $0.500 \pm 0.008$ & $0.492 \pm 0.004$ & $0.682 \pm 0.003$ & $0.213 \pm 0.005$ \\
1 & $0.500 \pm 0.009$ & $0.500 \pm 0.011$ & $0.524 \pm 0.003$ & $0.286 \pm 0.007$ \\
\hline
\end{tabular}

\section{Conclusion}

In this paper, we have proposed a new simulation method for the artificial generation of classifier outputs to examine the relationship between the plurality voting performance and between-classifier diversity. An algorithm for building two classifiers with specified accuracies and a pair-wise agreement $\kappa$ between them is presented. The algorithm for generating more than 2 classifiers has also been proposed. The experimental results point out the advantages of plurality voting over majority voting when 
classifiers with different agreement levels are combined. The proposed method will be useful to evaluate any abstract-level combination methods and thus will be helpful to clarify the conditions under which a combination method can be used or is the best for different pattern recognition problems.

\section{References}

1. L. Breiman, Bagging predictors, Machine Learning, 24:2, (1996), 123-140.

2. R.P. Duin, The combining classifier: to train or not to train?, in: R. Kasturi, D. Laurendeau, C. Suen (eds.), ICPR16, Proceedings $16^{\text {th }}$ International Conference on Pattern Recognition, Canada, vol. II, IEEE Computer Society Press, Los Alamitos, (2002), 765-770.

3. J. Fleiss, Statistical methods for rates and proportions, John Wily \& sons, (1981).

4. G. Giacinto, F. Roli, Design of effective neural network ensembles for image classification processes, Image Vision and Computing Journal, 19:9/10, (2001), 699-707.

5. L. Hansen, P. Salamon, Neural network ensembles, IEEE Transactions on Pattern Analysis and Machine Intelligence, 12, (1990), 993-1001.

6. J. Kittler, M. Hatef, R.P.W. Duin, J. Matas, On combining classifiers, IEEE Transactions on Pattern Analysis and Machine Intelligence, 20:3, (1998).

7. L.I. Kuncheva, C.J. Whitaker, C.A. Shipp, R.P.W. Duin, Is independence good for combining classifiers ?, ICPRI 5, Proceedings $15^{\text {th }}$ International Conference on Pattern Recognition, 2, (2000), 168-171.

8. L.I. Kuncheva, R.K Kountchev, Generating classifier outputs of fixed accuracy and diversity, Pattern Recognition Letters, 23. (2002), 593-600.

9. L.I. Kuncheva, C.J. Whitaker, Measures of diversity in classifier ensembles and their relationship with the ensemble accuracy, Machine Learning, 51, (2003), 181-207.

10. L.I. Kuncheva, That elusive diversity in classifier ensembles, Proc IbPRIA 2003, Mallorca, Spain, Lecture Notes in Computer Science, Springer-Verlag, (2003), 1126-1138.

11. L.I. Kuncheva, C.J. Whitaker, R.P.W. Duin, Limits on the majority vote accuracy in classifier fusion, Pattern Analysis and Applications, 6, (2003), 22-31.

12. L. Lam, C.Y. Suen, Application of majority voting to pattern recognition : an analysis of its behavior and performance, IEEE Transactions on System, Man, and Cybernetics, 27:5, (1997).

13. V.D. Lecce, G. Dimauro, A. Guerrierro, S. Impedovo, G. Pirlo, A. Salzo, Classifier combination: the role of a-priori knowledge, In Proceedings of the $7^{\text {th }}$ International Workshop on Frontiers in Handwriting Recognition, Amsterdam, The Netherlands (2000), 143-152.

14. X. Lin, S. Yacoub, J. Burns, S. Simske, Performance analysis of pattern combination by plurality voting, Pattern Recognition Letters, 24, (2003), 1959-1969.

15. D. Ruta, B. Gabrys, New measure of classifier dependency in multiple classifier systems, In Proceedings of the $3^{\text {rd }}$ International Workshop on Multiple Classifier System (MCS), Lecture Notes Computer Science 2364, Calgary, Sardinia, Italy, (2002), 127-136.

16. D.M.J. Tax, M.V. Breukelen, R.P.W. Duin, J. Kittler, Combining multiple classifiers by averaging or by multiplying ?, Pattern Recognition, 33, (2000), 1475-1485.

17. M. VanErp, An overview and comparison of voting methods for pattern recognition, International Workshop Frontiers Handwritten Recognition, (2002), 195-200.

18. H. Zouari, L. Heutte, Y. Lecourtier, A. Alimi, Simulating classifier outputs for evaluating parallel combination method, Lecture Notes in Computer Science, $4^{\text {th }}$ International Workshop, Multiple Classifier Systems (MCS), Guildford, UK, (2003), 296-305. 